\title{
Premio Fundación Dr. Pedro Cossio 2020
}

\author{
2020 Dr. Pedro Cossio Foundation Award
}

JORGE LERMAN

Presidente del Jurado

La versión número 45 del histórico Congreso de nuestra Sociedad Argentina de Cardiología se llevó a cabo del 19 al 21 de noviembre de 2020. Como casi todo lo que ocurrió durante este año tan especial, fue una experiencia totalmente novedosa. Los Comités Organizador y Científico debieron reinventarse y transformar este enorme evento de presencial en virtual mediante las diversas herramientas proporcionadas por la tecnología informática. En lugar de contar con el contacto directo con los presentadores de los trabajos, el Jurado accedió a su versión grabada, y no pudo existir el aporte de comentadores expertos en los diversos ejes temáticos.

El Comité Científico del Congreso seleccionó 6 trabajos como candidatos para ganar el Premio Fundación Dr. Pedro Cossio 2020. De acuerdo a lo acostumbrado desde hace 32 años, haremos breves comentarios acerca de estos.

Resultó ganador el siguiente trabajo:

Asociación entre interleuquinas inflamatorias y la presencia de trastornos intraventriculares de la conducción en pacientes con serología positiva para enfermedad de Chagas y función ventricular conservada.

Dres. Mario Principato, Analia Paolucci, Silvia Miranda, Gustavo Sosa. TPC Maria Von Vulffen, Alejandro Tomatti, Guillermo Di Girolamo, Justo Carbajales

Además del daño directo provocado por el parásito, existe evidencia de actividad inmunológica e inflamatoria en la fisiopatología de la enfermedad de Chagas (ECh). Hace 40 años, el Dr. Patricio Cossio, uno de los hijos del prestigioso cardiólogo al que se dedica este premio de la Sociedad Argentina de Cardiología efectuó estudios pioneros en este campo. (1)

Más recientemente, se comprobó que anticuerpos IgG contra receptores muscarínicos M2 son responsables de bradiarritmias y trastornos de la conducción aurículo-ventricular. (2) Autoanticuerpos contra receptores $\beta 1$ y $\beta 2$ pueden ser producidos por pacientes (p) con ECh como fue ya demostrado por autores del grupo que desarrolló este trabajo. $(3,4)$ Estos autoanticuerpos, originalmente dirigidos contra el parásito, reconocerían antígenos del huésped. Asimismo se sabe que las citoquinas inflamatorias agreden al tejido miocárdico y provocan disfunción ventricular en esta enfermedad. (5)

El propósito de este estudio realizado en el Hospital Ramos Mejía de Buenos Aires y el Instituto Alberto C Taquini de Investigaciones en Medicina Traslacional fue indagar si la inflamación sistémica podría estar relacionada con la aparición de trastornos de conducción intraventricular (TCIV) en etapas precoces de la ECh. Se incluyeron 22 p entre 21 y 80 años, seropositivos para ECh con función ventricular izquierda igual o mayor del $50 \%$. Se constituyó, además, un grupo control de 14 individuos sanos de similar edad e igual proporción de sexos, con serología negativa para ECh. Se midió la concentración plasmática de varios marcadores inflamatorios y se analizó la presencia de TCIV: bloqueo de rama derecha, bloqueo de rama izquierda o hemibloqueo izquierdo anterior. Diez de los $22 \mathrm{p}$ seropositivos tuvieron TCIV. Estos mostraron valores significativamente más elevados de interleuquinas $10,2,12 \mathrm{p} 70,15, \mathrm{INF} \gamma$ y MP1 $\alpha$ que los 12 que no los tuvieron. Los p con TCIV eran mayores $(61,3 \pm$ 13,3 vs 50,9 $\pm 6,2$ años) y su fracción de eyección del ventrículo izquierdo algo más baja $(59,6 \% \pm 6,2$ vs. $62 \% \pm 7,0)$ que aquellos sin TCIV. Estas diferencias no fueron significativas probablemente por el número reducido de la muestra. Por otra parte, los p sin TCIV mostraron concentraciones de citoquinas similares a los controles. Estos resultados indican un hallazgo novedoso: $\mathrm{p}$ chagásicos con función ventricular preservada, pero con TCIV tienen aumento de citoquinas proinflamatorias, hecho que debería ser reconfirmado en estudios más amplios.

Los demás trabajos presentados fueron los siguientes:

Lipoproteína (a) en población general con riesgo cardiovascular moderado y colesterol LDL aumentado ¿Cuál es la prevalencia y asociación con el antecedente de enfermedad cardiovascular prematura?

Dres. Pablo Gulayin, Alfredo Lozada, Laura Schreier, Laura Gutiérrez, Graciela López, Matías Calandrelli, Fernando Lanas, Vilma Irazola.

Este nuevo aporte del Centro de Excelencia en Salud Cardiovascular para el Cono Sur (CESCAS) se suma a otros trabajos del mismo grupo, que habían sido seleccionados en anteriores versiones del presente premio y al que fuera ganador en 2017.

La lipoproteína (a) o LP(a) está compuesta por una molécula de lipoproteína LDL rica en colesterol asociada por puentes disulfuro a una molécula de apolipoproteína (a). Esta última posee una estructura similar al plasminógeno, al que interfiere como inhibidor competitivo y, en consecuencia, bloquea su propiedad 
fibrinolítica. Esto le confiere capacidad pro trombótica y antifibrinolítica. Además, la LP(a) puede atravesar fácilmente el endotelio y contribuir a la formación y crecimiento de las placas de ateroma. Este doble mecanismo hace de la LP(a) un nuevo factor de riesgo de enfermedad ateroesclerótica, particularmente en el territorio coronario, que no está aún considerado en los diferentes índices (scores) de riesgo conocidos. (6)

Este trabajo tuvo dos objetivos: evaluar la prevalencia de $\mathrm{LP}(\mathrm{a})$ elevada (mayor de $125 \mathrm{nmol} / \mathrm{L}$ ) en población general con riesgo cardiovascular moderado (entre 10 y $20 \%$ ) y LDL colesterol elevado (mayor de $130 \mathrm{mg} / \mathrm{dL}$ ); y determinar la asociación entre antecedentes familiares de enfermedad cardiovascular prematura (AFECVP) y LP(a) elevada en habitantes de 4 ciudades del Cono Sur de América Latina: Marcos Paz y Bariloche (Argentina), Canelones (Uruguay) y Temuco (Chile). La muestra consistió de 7524 individuos (2165 varones) entre 35 y 74 años. Del total de la población, alrededor del $11 \%$ tenía antecedentes de AFECVP y el 6,1\% tenían LP(a) elevada. El hallazgo más trascendente de este estudio fue que entre los individuos con LDL colesterol $\geq 160 \mathrm{mg} / \mathrm{dL}$, la LP(a) estaba elevada en $5 \%$ de los casos sin AFECVP, pero en $18 \%$ de aquellos con AFCVP $(p=0,026)$. Este trabajo confirma la fuerte asociación genética de la elevación de la LP(a) y el hecho de que una simple pregunta acerca de la historia familiar en los p con elevado LDL colesterol podría contribuir a descubrir individuos con elevación de este nuevo factor de riesgo no muy comúnmente investigado, pero de fuerte impacto en la historia natural y el pronóstico de la enfermedad ateroesclerótica.

El hiperaldosteronismo se encuentra relacionado con factores pronósticos en pacientes con hipertensión pulmonar en buena clase funcional.

Dres. Andrés Atamañuk, Jorge Zambrano, Ignacio Gandino, Diego Hoffmann, Rafael Zaug Nadur, Sebastián Obregón, Luis Gómez, Diego Litewka.

$\mathrm{El}$ sistema renina-angiotensina-aldosterona (SRAA) es uno de los mecanismos humorales básicos en la generación y la progresión de la hipertensión arterial sistémica y la insuficiencia cardíaca por diversos mecanismos. Entre los más importantes y conocidos figuran la vasoconstricción con aumento de la poscarga, la retención de sodio con aumento de la precarga, el remodelado vascular y del ventrículo izquierdo. La importancia terapéutica derivada de su inhibición es uno de los pilares de la terapéutica de esas condiciones. (7) La activación del SRAA en hipertensión pulmonar (HP) es menos explorada, pero podría tener implicancias pronósticas. (8)

Los autores del presente trabajo, del grupo del Hospital Fernández de Buenos Aires, efectuaron un estudio prospectivo y observacional de p con HP diagnosticada por cateterismo cardíaco derecho. Analizaron 38 p (36 eran mujeres) con HP estable en clase funcional (CF) I o II. Midieron aldosterona plasmática (AP), actividad de renina plasmática (ARP) y calcularon el índice $\mathrm{AP} /$
ARP. El objetivo fue identificar la presencia de hiperaldosteronismo (HA) en HP y su implicancia pronóstica. Se definió HA como ARP $<1 \mathrm{ng} / \mathrm{mL} / \mathrm{h}$ y AP/ARP $>20$; AP elevada, $>90 \mathrm{pg} / \mathrm{mL}$; y ARP baja, $<1 \mathrm{ng} / \mathrm{mL} / \mathrm{h}$. Además, estudiaron la distancia alcanzada en la prueba de caminata de seis minutos (TC6M) y la concentración de NT-proBNP, como factores pronósticos.

Los p con HA confirmaron tener menor ARP: 0,29 $\pm 0,01$ vs. $1,26 \pm 1,1(p=0,043)$ y mayor AP/ARP: $35,9 \pm 11,8$ vs. $8,9 \pm 5,0(p=0,001)$. Además, menor distancia caminada en el TC6M: $343,5 \pm 101$ vs. 445,6 $\pm 83 \mathrm{~m}(p=0,012)$ y NT-proBNP más elevado: 1602,2 \pm 1492 vs. $562,7 \pm 854 \mathrm{pg} / \mathrm{mL}(p=0,022)$. Estos son signos indirectos que sugieren que p con HP, CF no muy avanzada y HA tendrían peor pronóstico que el resto. Estos hallazgos preliminares deberían ser corroborados en un estudio más extenso, con un grupo control sin HA y seguimiento alejado.

¿La troponina de alta sensibilidad agrega valor pronóstico a los puntajes de riesgo validados para predecir mortalidad hospitalaria en pacientes con insuficiencia cardíaca aguda?

Lucrecia Burgos, Lorena Villalba, Rita Miranda, Andreína Gil Ramírez, Luján Talavera, Adriana Acosta, Fernando Botto, Mirta Diez.

La troponina T de alta sensibilidad (TnT-AS) es un marcador de amplia utilidad en las guardias hospitalarias y en las unidades coronarias para el diagnóstico de los síndromes coronarios agudos. En cambio, es escasa la evidencia acerca de su rol diagnóstico y pronóstico en la insuficiencia cardíaca aguda descompensada (ICAD).

El propósito de este trabajo fue determinar si la adición de TnT-AS a los puntajes de riesgo clínico mejora la predicción de mortalidad hospitalaria en pacientes con ICAD. Se realizó un análisis retrospectivo de una cohorte prospectiva y consecutiva proveniente del Instituto Cardiovascular de Buenos Aires. Se incluyeron 712 pacientes adultos internados entre 2015 y 2019 con diagnóstico primario de ICAD. La media de edad fue de $74,8 \pm 12,5$ años y el $64,1 \%$ eran hombres. El 47,8\% presentaban reducción de la fracción de eyección del ventrículo izquierdo (FEVI) $(<40 \%)$. Las causas más frecuentes de descompensación fueron transgresión alimentaria, infección y taquiarritmia supraventricular. Se calcularon tres puntajes de riesgo validados y ampliamente utilizados: OPTIMIZE-HF, GWTG-HF y ADHERE y se midió TnT-AS de ingreso al 79\% de la población. En el 91\% estaba elevada. El punto final primario fue mortalidad hospitalaria. Se realizó un subanálisis según la FEVI. La discriminación se evaluó mediante el área bajo la curva ROC (AUC-ROC).

La mortalidad hospitalaria fue del 8,7\%. El AUCROC (IC95\%) para TnT-AS aislada fue de 0,70 $(0,63$ $0,77)$ y de $0,80(0,74-0,87), 0,79(0,72-0,86)$ y 0,79 $(0,71-0,86)$ para el puntaje OPTIMIZE-HF, GWTG-HF y ADHERE, respectivamente. La adición de la TnT-AS a los modelos no aumentó el AUC-ROC. En el subanálisis, no se encontraron diferencias en los puntajes de riesgo con la adición de TnT-AS en la población 
con FEVI <40\% y $\geq 40 \%$. En conclusión, la TnT-AS elevada al ingreso fue frecuente y su incorporación a los puntajes de riesgo validados no determinó un beneficio pronóstico incremental en estos pacientes. Probables interpretaciones de estos resultados podrían ser la elevada prevalencia de TnT-AS en la población analizada y la relativa baja frecuencia de la etiología isquémica en ella, dado que la TnT-AS es básicamente un marcador de necrosis y, entre estos pacientes, la etiología isquémica no fue de las más frecuentes.

Evolución clínica y pronóstico de una población de pacientes con miocardiopatía hipertrófica no obstructiva.

María Banchio Dal Bo, Nahuel Messina, Alejandro Quiroga, Guido Antoniutti, José Horacio Casabé, Juan Mouzarkel, Luis Verón, Adrián Fernández

La mayoría de los p con miocardiopatía hipertrófica pertenecen a la forma obstructiva ( $70 \%)$, definida como la presencia de un gradiente de presión al nivel del tracto de salida del ventrículo izquierdo igual o mayor de $30 \mathrm{mmHg}$, ya sea en reposo o provocable mediante la maniobra de Valsalva o posterior al esfuerzo físico. La forma no obstructiva (MPHn) (30\%) puede transcurrir durante prolongados períodos de estabilidad clínica con relativamente escaso riesgo de insuficiencia cardíaca (IC) o arritmias graves. (9)

Este grupo de la Fundación Favaloro efectuó un estudio retrospectivo y observacional de 225 p consecutivos con MPHn (69\% varones) con edad promedio de $45 \pm 16$ años seguidos entre 1993 y 2020 . Los puntos finales primarios fueron muerte súbita (MS) o descarga apropiada por un cardiodesfibrilador implantable (CDI), considerado equivalente de MS, y muerte por IC o requerimiento de trasplante cardíaco, considerado equivalente de muerte por IC. El punto final secundario fue desarrollo de fibrilación auricular. Cinco $\mathrm{p}$ (3\%) presentaban insuficiencia mitral grave. Pese a la ausencia de obstrucción significativa, 22 p (14\%) presentaron movimiento anterior sistólico de la valva anterior mitral, lo que pone en evidencia que existen múltiples mecanismos que lo generan y su presencia no se puede atribuir únicamente al gradiente obstructivo. Luego de un seguimiento medio de 7,5 años, $17 \mathrm{p}(7,6 \%)$ presentaron el punto final primario y 69 (31\%), el secundario. Por otro lado, 9 p (4\%) progresaron con deterioro de la FEVI en el seguimiento y $4(2 \%)$ requirieron trasplante cardíaco. A 50 p (22\%) se les indicó la colocación de un CDI. Este trabajo es una interesante observación que ilustra acerca de las características ecocardiográficas y evolución clínica de p con MPHn. A pesar de que en general se la considera una afección relativamente benigna, no se pueden descartar complicaciones a mediano plazo. Esto enfatiza que estos p deben tener un seguimiento apropiado. La detección de fibrosis mediante realce tardío en la resonancia magnética contribuye a individualizar $\mathrm{p}$ de mayor riesgo y el estudio genético a identificar grupos familiares.
Cometas pulmonares en ecografía pulmonar ¿̇Un mejor concepto de congestión y variabilidad en insuficiencia cardiaca?

Lilia Princich, Nadia Vergara, Ezequiel Besmalinovich, Agustina Rossi, Paul Vargas Mieles, Christian Musante, Romina Caprini, Adrián Lescano

La ecografía pulmonar (EP) en insuficiencia cardíaca (IC) es un recurso diagnóstico de utilidad para la detección y evaluación de derrame pleural. Otra indicación es la determinación de la congestión vascular pulmonar, líquido extravascular alvéolo intersticial, y la respuesta al tratamiento mediante la observación de los "cometas pulmonares" (CP). Sin embargo, esta última indicación es escasamente utilizada: en el registro ARGEN-IC, su empleo fue del 9\%. (10) Los CP son equivalentes a las líneas B de Kerley de la radiografía de tórax y se examinan ubicando el transductor del ecocardiógrafo perpendicular al tórax. Se ven como líneas angostas, densas, que se inician en la línea pleural y llegan hasta el final de la pantalla, son dinámicas y se mueven con el deslizamiento pleural.

El presente trabajo del Sanatorio de la Trinidad de Quilmes consistió en el análisis de 49 p con IC, 73,4\% de sexo masculino, y edad media de $70 \pm 14$ años. El $80 \%$ tenía hipertensión arterial, $46 \%$ diabetes, $25 \%$ historia de infarto de miocardio, $23 \%$ valvulopatía significativa y $36 \%$ fibrilación auricular. La forma de presentación de IC al ingreso fue: el $29 \%$, congestión pulmonar; el $11 \%$, congestión periférica; el $44 \%$ congestión mixta; y el $16 \%$ edema agudo de pulmón. El 58\% tuvo FEVI deteriorada, el $20 \%$ intermedia y el $22 \%$ preservada. El $91 \%$ tuvo CP en la ecografía pulmonar, con una media de 27,3 $\pm 11,17$. Cuando se comparó la diferencia entre el ingreso y el alta se observó reducción significativa de la cantidad de CP: $27,3 \pm 11,17$ vs. $5,27 \pm 4,14$ ( $p<0,000)$. En cambio, no hubo cambios significativos en la FEVI, dimensiones ecocardiográficas, relación E/e' del flujo transmitral ni en el pequeño subgrupo (23\% de los casos) en el que se midió NT-proBNP. Los autores concluyen que los CP se observan en una alta proporción de $\mathrm{p}$ con insuficiencia cardíaca y, a pesar de lo reducido de la muestra, fue el único marcador que demostró variabilidad significativa asociada al tratamiento durante la internación. Cuando un paciente se interna con IC aguda y recibe el tratamiento correspondiente durante la hospitalización, en el momento del alta puede surgir la duda acerca de la continuación o no de los diuréticos y sus dosis. La investigación de los CP permite disponer de una aproximación objetiva, disponible y sencilla para determinar en forma seriada la gravedad de la congestión pulmonar y evaluar la efectividad del tratamiento.

Completaron el Jurado del Premio Fundación Dr. Pedro Cossio 2020 los Expresidentes de la Sociedad Argentina de Cardiología, Dres. Miguel González y Osvaldo Masoli, a quienes agradezco su participación capacitada y responsable. 


\section{Bibliografía}

1. Cossio PM, Diez C, Laguens RP, Arana RM. Inmunopatología de la Enfermedad de Chagas. Medicina (Buenos Aires) 1980; 40 (Supl. 1): 222-30. 2. Thiers C, Barbosa J, Pereira B, Nascimento E, Nascimento J, Medei $\mathrm{E}$, et al. Autonomic dysfunction and anti-M2 and anti- $\beta 1$ receptor antibodies in Chagas disease patients. Arq Bras Cardiol. 2012;99:732-9. https://doi.org/10.1590/S0066-782X2012005000067

3. Rosenbaum M B, Chiale P A, Schejtman D, Levin M, Elizari M V. Antibodies to Beta-Adrenergic Receptors Disclosing Agonist-Like Properties in Idiopathic Dilated Cardiomyopathy and Chagas' Heart Disease. J Cardiovascular Electrophysiol 1994;5:367-75. https://doi. org/10.1111/j.1540-8167.1994.tb01174.x

4. Chiale P A, Rosenbaum M B, Elizari M V, Hjalmarson A, Magnusson Y, Wallukat G, et al. High Prevalence of Antibodies against Beta 1- and Beta-Adrenoceptors in Patients with Primary Electrical Cardiac Abnormalities. J Am Coll Cardiol 1995;26:864-9. https://doi. org/10.1016/0735-1097(95)00262-2

5. Rodrigo Sousa G. Plasma Cytokine Expression Is Associated with Cardiac Morbidity in Chagas Disease. PLoS One. 2014;9(3): e87082. https://doi.org/10.1371/journal.pone.0087082
6. Nordestgaard BG, Langsted A. Lipoprotein (a) as a cause of cardiovascular disease: insights from epidemiology, genetics, and biology. Journal of lipid research 2016;57:1953-75. https://doi.org/10.1194/ jlr.R071233

7. Pitt B, Zannad F, Remme WJ, Cody R, Castaigne A, Perez A, et al. The effect of spironolactone on morbidity and mortality in patients with severe heart failure. N Engl J Med. 1999;341:709-17. https:// doi.org/10.1056/NEJM199909023411001

8. Maron BA, Leopold JA. The role of the renin-angiotensin-aldosterone system in the pathobiology of pulmonary arterial hypertension (2013 Grover Conference series). Pulm Circ. 2014;4:200-10. https:// doi.org/10.1086/675984

9. Maron BJ, Rowin EJ, Maron MS, Braunwald E. Nonobstructive Hypertrophic Cardiomyopathy Out of the Shadows: Known from the Beginning but Largely Ignored ... Until Now. Am J Med. 2017; 130:119-23. https://doi.org/10.1016/j. amjmed.2016.09.015

10. Lescano A, Soracio G, Soricetti J, Arakaki D, Coronel L, Cáceres L et al. Registro Argentino de Insuficiencia Cardíaca Aguda (Argen IC). Evaluación de cohorte parcial de 30 días. Rev Argent Cardiol 2020;88:118-25. https://doi.org/10.7775/rac.es.v88.i2.17201 